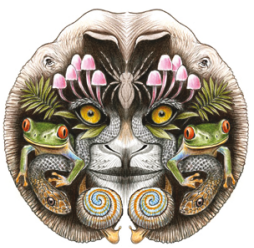

ISSN

Online 0974-7907

Print 0974-7893

OPEN ACCESS

\title{
SUbSPECIES IDENTIFICATION OF CHIMPANZEES PAN tROglodytes (PRIMATES: HOMINIDAE) fROM THE NATIONAL ZOO OF THE Metropolitan Park of Santiago, Chile, using MITOCHONDRIAL DNA SEQUENCES
}

\author{
Javier Andrés Vega ${ }^{1}$, José Suazo ${ }^{2}$, Susan Valerie Smalley ${ }^{3}$, Luis Rodrigo Cataldo ${ }^{4}$, \\ Guillermo Cubillos ${ }^{5} \&$ José Luis Santos ${ }^{6}$ \\ ${ }_{1,3,4,6}$ Department of Nutrition, Diabetes and Metabolism, School of Medicine, \\ Pontificia Universidad Católica de Chile, Santiago, Chile \\ ${ }^{2}$ Instituto de Investigación en Ciencias Odontológicas, Facultad de Odontología, Universidad de Chile, Santiago, Chile. \\ ${ }^{5}$ Animal Welfare and Behavior Section, Zoológico Nacional del Parque Metropolitano de Santiago, Chile \\ ${ }^{1}$ jnvega@uc.cl, ${ }^{2}$ jsuazo@odontologia.uchile.cl, ${ }^{3}$ svsmalleym@gmail.com, ${ }^{4}$ rcataldo@ciq.uchile.cl, \\ ${ }^{5}$ gcubillos@parquemet.cl, ${ }^{6}$ jsantos@med.puc.cl (corresponding author)
}

Abstract: Natural populations of Chimpanzees Pan troglodytes are declining because of hunting and illegal live animal trafficking. Four subspecies of Chimpanzee have been reported: Pan troglodytes troglodytes, P.t. schweinfurthii, P.t. verus and P.t. ellioti, which have remained geographically separated by natural barriers such as the rivers Niger, Sanaga and Ubangi in central Africa. Sequence analysis of mitochondrial DNA (mtDNA) has been used for the determination of these subspecies, which indirectly can also suggest their geographic origin. It was decided to identify the subspecies and the geographic origin of three captive chimpanzees of the National Zoo of the Metropolitan Park of Santiago (Chile), by analyzing their mitochondrial DNA. DNA was extracted from the saliva of three adult chimpanzees (two males and one female). After the analysis of sequences of the mitochondrial hypervariable region (HVI), a phylogenetic tree was constructed using mitochondrial sequences of known Pan troglodytes subspecies. Molecular phylogeny analysis revealed that the chimpanzees are likely to belong to three different subspecies: P.t. schweinfurthii, P.t. verus and P.t. troglodytes. Identification of subspecies of the three chimpanzees of the National Zoo of the Metropolitan Park of Santiago (Chile) was possible due to mtDNA analysis. Future identification of chimpanzees will allow the development of a studbook for the chimpanzee subspecies in other Latin American zoos.

Keywords: Genetic distance, geographical localization, Hypervariable region.
Natural populations of Chimpanzees Pan troglodytes are declining due to hunting and illegal live animal trafficking (Oates 2006). The Chimpanzee is as an Endangered species according to the IUCN Redlist (Oates et al. 2013).

Latin American zoos host many wild-animal colonies (http://www.alpza.com), however, identification upon arrival of any new organism has been made only from the phenotypic characteristics like color pattern (feathers, plates, fur, scales), body size or dental formula (Klukkert et al. 2012). In addition, the exact geographical origin of many animals in captivity is usually unknown and the case of Chimpanzees in Latin American zoos are no exception to this rule. Currently, Chimpanzees are known by four phenotipically recognized subspecies: P.t. troglodytes, P.t. schweinfurthii, P.t. verus and P.t. ellioti (formerly called P.t. vellerosus) (Groves 2001; Oates et al. 2009). Interestingly, these four subspecies have different geographic origins that have remained spatially

DOI: http://dx.doi.org/10.11609/JoTT.o3611.5712-7 | ZooBank: urn:Isid:zoobank.org:pub:FBCECF51-1A84-4FBD-85AF-1E8F0811E511

Editor: Mandar Paingankar, University of Pune, Pune, India.

Date of publication: 26 May 2014 (online \& print)

Manuscript details: Ms \# 03611 | Received 03 May 2013 | Final received 04 April 2014 | Finally accepted 07 April 2014

Citation: Vega, J.A., J. Suazo, S.V. Smalley, L.R. Cataldo, G. Cubillos \& J.L. Santos (2014). Subspecies identification of Chimpanzees Pan troglodytes (Primates: Hominidae) from the National Zoo of the Metropolitan Park of Santiago, Chile, using mitochondrial DNA sequences. Journal of Threatened Taxa 6(5): 5712-5717; http://dx.doi.org/10.11609/JoTT.03611.5712-7

Copyright: (C) Vega et al. 2014. Creative Commons Attribution 4.0 International License. JoTT allows unrestricted use of this article in any medium, reproduction and distribution by providing adequate credit to the authors and the source of publication.

Funding: Supported by VRAID 02/2009 project of the Pontificia Universidad Católica de Chile.

Competing Interest: The authors declare no competing interests.

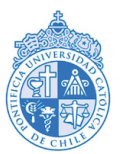

Acknowledgements: We thank the staff of the Metropolitan Park of Santiago for their support in obtaining saliva samples. Primer sequences for mtDNA analysis were kindly provided by Prof. George Perry (Anthropological Genomics Laboratory, Penn State University). 


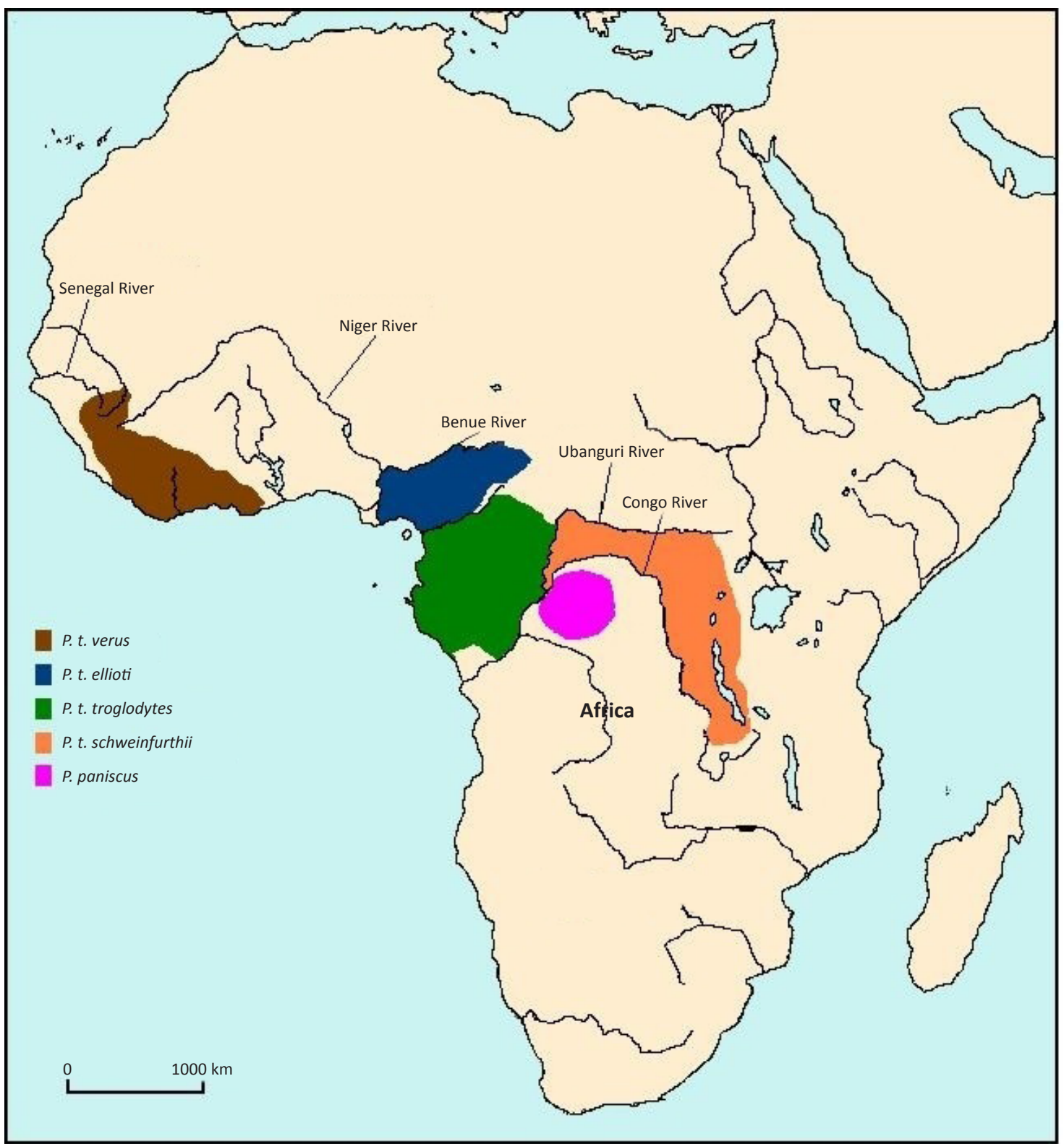

Figure 1. Geographical distribution of different subspecies of Pan troglodytes and Pan paniscus in Africa.

Geographical distribution location of the four subspecies of Chimpanzee and Pan Paniscus, represented with a different color in the map.

separated during the course of evolution due to natural barriers of central Africa, such as the rivers Niger, Sanaga and Ubangi (Kortlandt 1983) (Fig. 1).

Sequence analysis of mitochondrial DNA (mtDNA) in the hypervariable region $\mathrm{HVI}$ is informative for the determination of Chimpanzee subspecies and also indirectly allows inference of their geographic origin (Goldberg \& Ruvolo 1997; Excoffier et al. 1999; Ely et al. 2005; Stone et al. 2010). Following this approach, subspecies of chimpanzees can be divided into Western (P.t. verus and P.t. ellioti), Central (P.t. troglodytes) and Eastern Chimpanzees (P. t. schweinfurthii) (Gagneux
2002).

The aim of this study was to identify the subspecies and the geographic origin of three captive chimpanzees of the National Zoo of the Metropolitan Park of Santiago (Chile), by analyzing their mitochondrial DNA.

\section{Material and Methods}

Ethics statement: The study was approved by the National Zoo of the Metropolitan Park of Santiago (Chile) following the ethical criteria of The Latin American Association of Zoological Parks and Aquariums (ALPZA).

Biological samples and laboratory methods: 
Mitochondrial DNA was extracted from saliva of three adult captive Chimpanzees (Pan troglodytes) of the National Zoo of the Metropolitan Park of Santiago using the Oragene Animal Kit (DNA Genotek, Canada) following the manufacturer's instructions. Specimen 1 (died in 2010) and specimen 2 were males with an estimated ages of 30 and 12 years respectively, while specimen 3 was a 35 years female. The study was conducted during the year 2010.

A total of 100ng of DNA was amplified by Polymerase Chain Reaction (PCR) with primers obtained in literature 5'-CTCTGTTCTTTCATGGGGAAGC-3' (forward) and 5'-CGGGATATTGATTTCACGGAGG-3' (reverse) (Goldberg \& Ruvolo 1997; Perry et al. 2012). Initially, the DNA was heated to $94^{\circ} \mathrm{C}$ for five minutes, followed by 35 cycles of denaturing, annealing, and elongation $\left(94^{\circ} \mathrm{C}\right.$ for 30 seconds, $54^{\circ} \mathrm{C}$ for 30 seconds, and $72^{\circ} \mathrm{C}$ for 30 seconds). The final extension occurred at $72^{\circ} \mathrm{C}$ for 10 minutes. PCR products (388 base pairs) were visualized by $1.5 \%$ agarose gel electrophoresis. The purified PCR products were sequenced with the big dye terminator sequencing kit v3.1 (Applied Biosystems, USA), using forward and reverse primers in two independent reactions, and analyzed in the $A B I$ prism $3730 X \mathrm{~L}$ sequencer (Applied Biosystems, USA).

Data analysis: For the genetic distance analysis of mtDNA sequences, we compared 28 complete mitochondrial sequences of Pan troglodytes comprising the four chimpanzee subspecies retrieved from Genbank (http://www.ncbi.nih.gov/genbank/), one mitochondrial sequence of the related-species Bonobo (Pan paniscus) as an out-group sequence, and sequences of the three chimpanzees from the Santiago Zoo. The sequences were submitted to Genbank, with the following accession numbers: KJ606391, KJ606392 and KJ606393, for specimens 1 (Images 1,2), 2 (Image 3) and 3 (Images $4,5)$, respectively.

The similarity of our sequences with Genbank database entries was carried out using BLAST (http:// blast.ncbi.nlm.nih.gov). All 32 sequences were aligned and compared using the program CLUSTALW (http:// www.ebi.ac.uk/clustalw), with gaps and missing data eliminated from the dataset ("complete deletion option"). There were a total of 359 positions analyzed in the final dataset. Phylogenetic trees were constructed with the Program MEGA6.05 (Tamura et al. 2013) using the Maximum Likelihood method with the HasegawaKishino-Yano (HKY) model (lowest BIC score= 3846) and a gamma distribution (5 discrete gamma categories). (Posada \& Crandall 2001). All positions containing gaps and missing data were eliminated. The construction of the phylogenetic tree allows the placement of mitochondrial sequences according to their genetic similarity in comparison with Pan troglodytes mtDNA sequences of specimens of known subspecies. The branch support for the correct location of branches was assessed through 1,000 bootstrap replicates.

\section{Results}

Specimen 1 showed $98 \%$ similarity with P.t. schweinfurthii, specimen 2 showed 95\% similarity with P.t. verus and specimen 3 showed $98 \%$ similarity with P.t. troglodytes. Results of BLAST analysis suggest that the specimen 1 from the Santiago Zoo was closely related to P.t. schweinfurthii, specimen 2 was grouped with P.t. verus and specimen 3 was closely related to P.t. troglodytes. Nucleotide sequences of the three chimpanzees of the Metropolitan National Zoo Park of Santiago and 29 reference sequences of Chimpanzees from different geographical locations were used for the construction of a maximum likelihood phylogenetic tree (Fig. 2). The bootstrap values, showed in the nodes of the branches, within the different clusters of chimpanzees are relatively high. Therefore, it is likely that specimen 1 was of the subspecies P.t. schweinfurthii, the specimen 2 is likely to be P.t. verus and the specimen 3 (female) is likely to be P.t. troglodytes.

\section{Discussion}

The correct subspecies classification of Chimpanzees in captivity may contribute in the efforts to reintroduce and establish the captive population's management programs with individuals of known origin, minimizing the likelihood of deleterious genetic effects caused by inbreeding (Goldberg \& Ruvolo 1997). In this context, genetic marker studies with nuclear and mitochondrial DNA sequences show that the nucleotide diversity in chimpanzees is four times higher than in humans, which favors the use of mtDNA for identifying subspecies of chimpanzees (Stone et al. 2002). It is also worth mentioning that the extent of genetic differentiation among chimpanzee subspecies is similar to the one found in human populations (Fischer et al. 2006). In this study, we have successfully identified, with high probability, three chimpanzee specimens of the National Zoo of Santiago of Chile, illustrating the utility of mtDNA in genetic identification (Ely et al. 2005).

Wild animal colonies represent a valuable opportunity for the ex situ conservation of the species and provide an important source of information for animal management plans. Given the current situation of the populations of chimpanzees held in captivity, it 


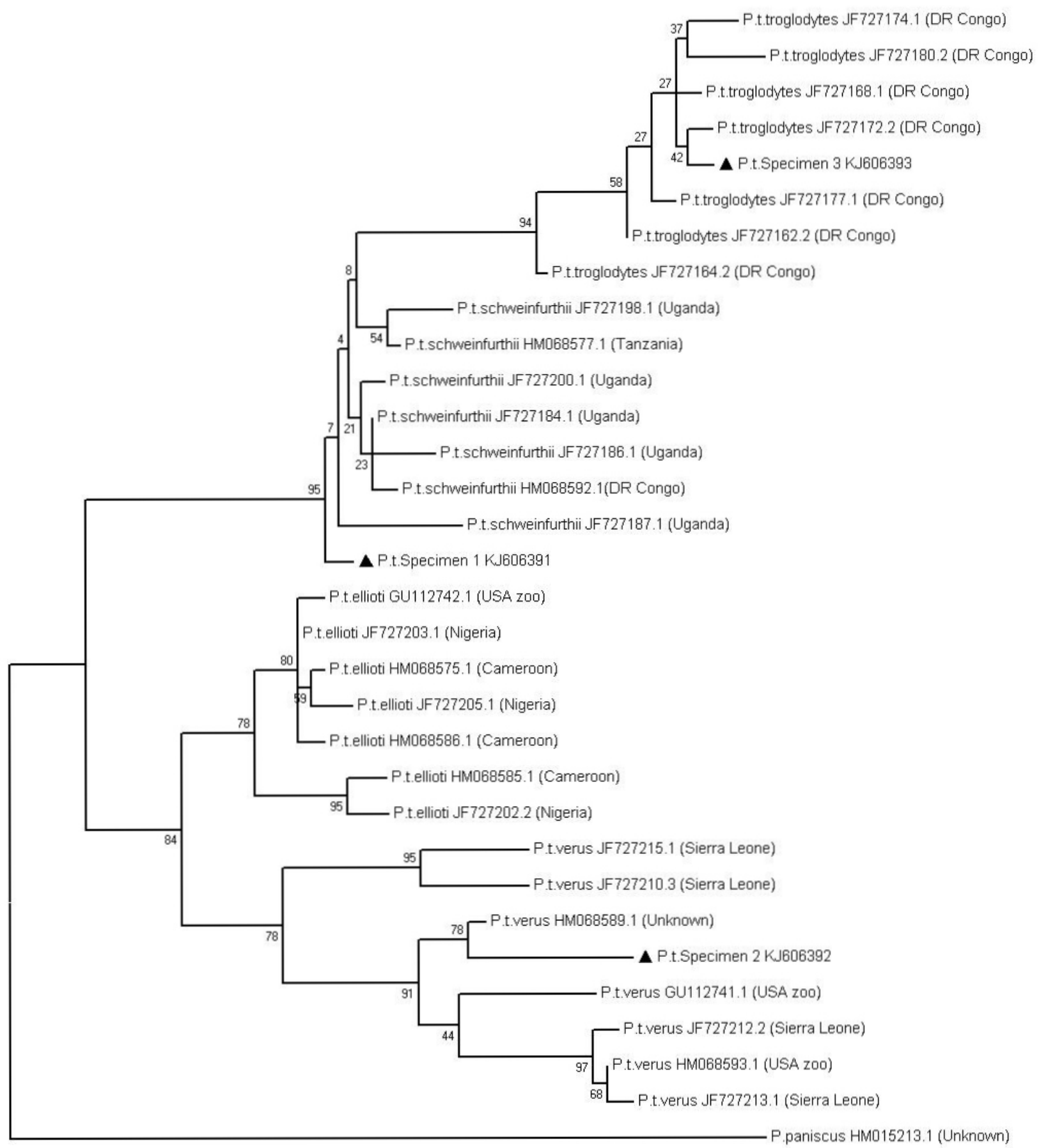

005

Figure 2. Phylogenetic relationship of Pan specimens.

A phylogenetic tree was constructed using the maximum likelihood method and the Hasegawa-Kishino-Yano (HKY) model with a gamma distribution. Nucleotide sequences of the three chimpanzees of the Metropolitan National Zoo Park of Santiago and 29 reference sequences of Chimpanzees from different geographical locations were used for molecular phylogeny analysis. Branches corresponding to partitions reproduced in less than $\mathbf{5 0 \%}$ bootstrap replicates are collapsed. The information of the sample origin of the chimpanzees was obtained from the literature and is shown in brackets. Bootstrap values are shown in each node. The Genbank number for sequences of current study is provided for each specimen. 


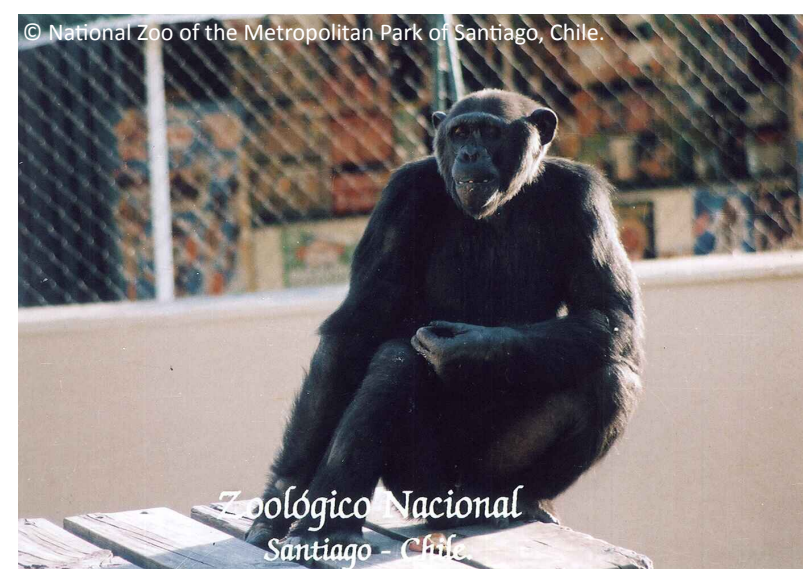

Image 1. Specimen 1 - Pan troglodytes schweinfurthii

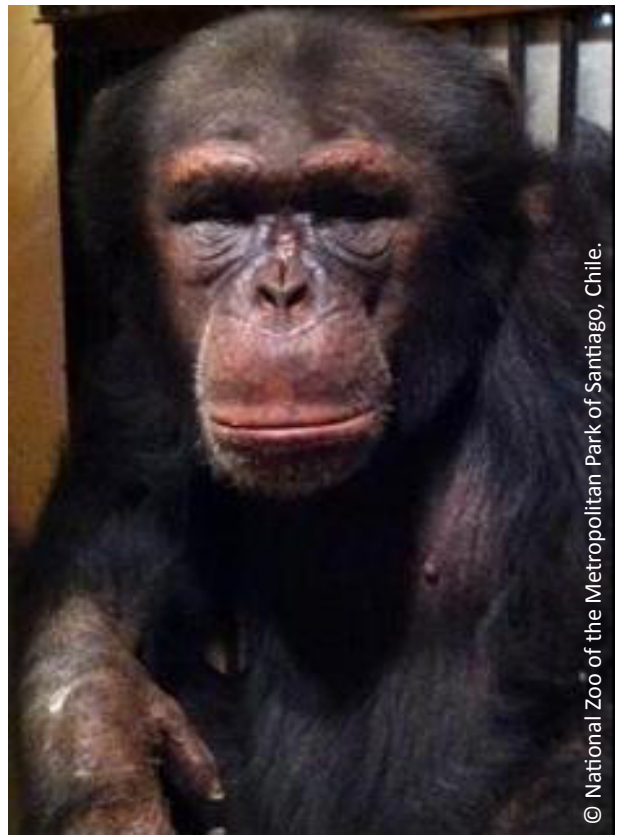

Image 3. Specimen 2- Pan troglodytes verus

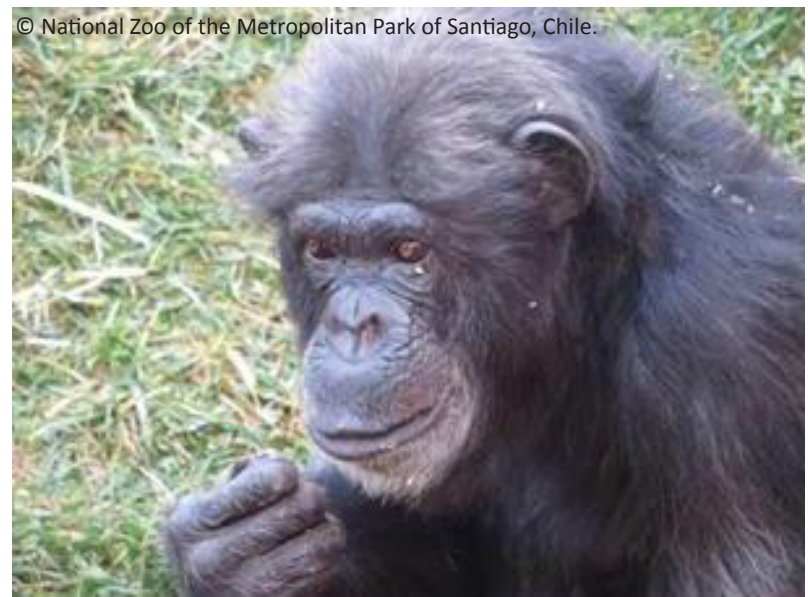

Image 5. Specimen 3- Pan troglodytes troglodytes

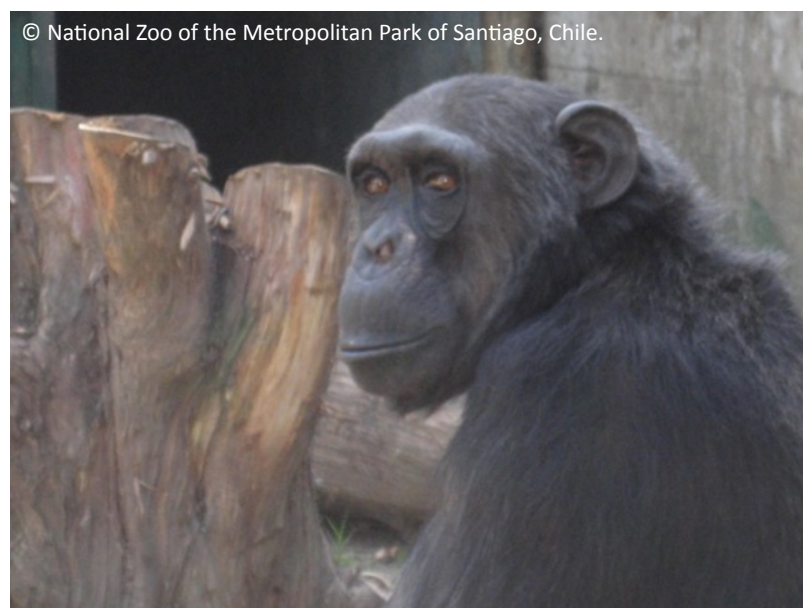

Image 2. Specimen 1 - Pan troglodytes schweinfurthii

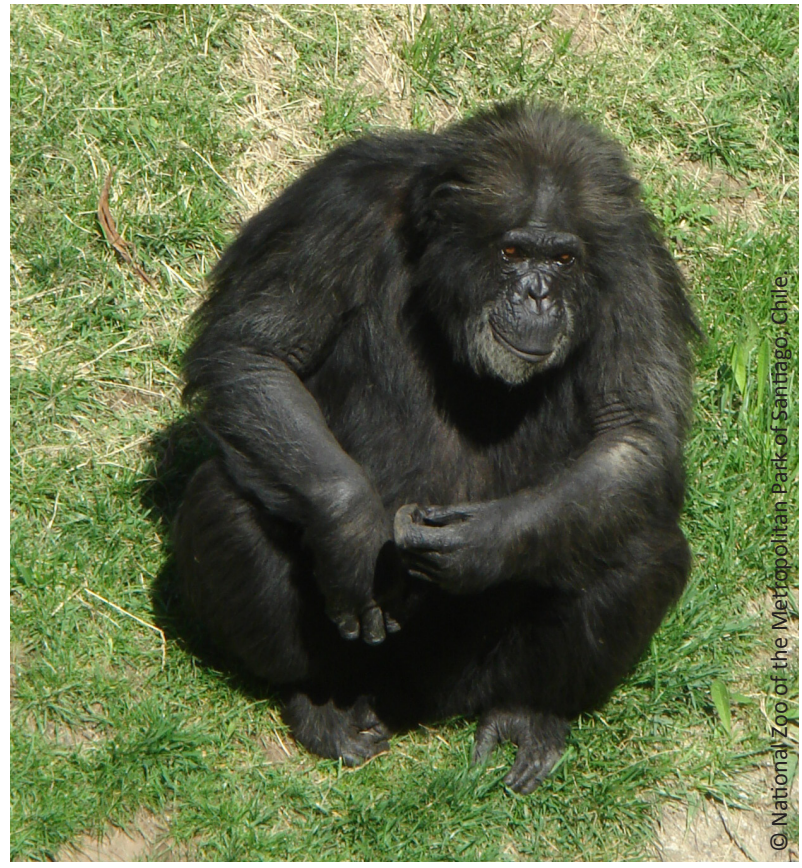

Image 4. Specimen 3 - Pan troglodytes troglodytes

is desirable to develop an identification plan involving Latin American zoological institutions. Zoos and primate rehabilitation centers could play a major role in the reception and subsequent reintroduction of animals, through development of studbooks for captive chimpanzees to minimize inbreeding and to preserve maximum genetic diversity (Carlsen 2009; Hvilsom et al. 2013). It should be noted that the release of captive chimpanzees in wild areas could be carried out successfully, representing a potential benefit to the conservation of this species (Goossens et al. 2005).

$A$ recent research on the genetic characterization of 
captive chimpanzees in Europe using 30 polymorphic microsatellite markers revealed that $40 \%$ of genotyped individuals were of western African origin, $18 \%$ of central Africa origin, $5 \%$ of eastern African origin and $23 \%$ hybrids (Hvilsom et al. 2013). A limitation of our study derives from the use of a single mitochondrial marker instead of nuclear polymorphic markers, which affects the accuracy of our subspecies classification.

In conclusion, identification of subspecies of the three chimpanzee specimens of the National Zoo of the Metropolitan Park of Santiago (Chile) was carried out by mtDNA analysis. A more elaborate genomic characterization with additional markers will facilitate the implementation of cooperative management plans and may constitute the initial steps for the creation of a studbook for chimpanzee subspecies of the region.

\section{References}

Carlsen, F. (2009). European Studbook for the Chimpanzee (Pan troglodytes). Copenhagen Zoo, Frederiksberg, Denmark.

Ely, J.J., B. Dye, W.I. Frels, J. Fritz, P. Gagneux, H.H. Khun, W.M Switzer \& D.R. Lee (2005). Subspecies composition and founder contribution of the captive U.S. Chimpanzee (Pan troglodytes) population. American Journal of Primatology 67(2): 223-241; http://dx.doi.org/10.1002/ajp.20179

Fischer, A., J. Pollack, O. Thalmann, B. Nickel \& S. Pääbo (2006). Demographic history and genetic differentiation in apes. Current Biology 16(11): 1133-1138; http://dx.doi.org/10.1016/j. cub.2006.04.033

Gagneux, P. (2002). The genus Pan: population genetics of an endangered outgroup. Trends in genetics 18(7)327-330; http:// dx.doi.org/10.1016/S0168-9525(02)02695-1

Goldberg, T.L. \& M. Ruvolo (1997). The geographic apportionment of mitochondrial genetic diversity in east African Chimpanzees, Pan troglodytes schweinfurthii. Molecular Biology and Evolution 14(9): 976-984; http://dx.doi.org/10.1093/oxfordjournals.molbev.a02584

Goossens, B., J.M. Setchell, E. Tchidongo, E. Dilambaka, C. Vidal, M. Ancrenaz \& A. Jamart (2005). Survival, interactions with conspecifics and reproduction in 37 chimpanzees released into the wild. Biological Conservation 123(4): 461-475; http://dx.doi. org/10.1016/j.biocon.2005.01.008

Groves, C.P. (2001). Primate Taxonomy. Smithsonian Institution Press, Washington, viii+350pp.
Hvilsom, C., P. Frandsen, C. Børsting, F. Carlsen, B. Sallé, B.T. Simonsen \& H.R Siegismund (2013). Understanding geographic origins and history of admixture among chimpanzees in European zoos, with implications for future breeding programmes. Heredity (Edinb) 110(6): 586-593; http://dx.doi.org/10.1038/hdy.2013.9

Kortlandt, A. (1983). Marginal habitats of chimpanzees. Journal of Human Evolution 12: 231-278; http://dx.doi.org/10.1016/S00472484(83)80149-3

Klukkert, Z.S., M.F. Teaford \& P.S. Ungar (2012). A dental topographic analysis of Chimpanzees. American Journal of Physical Anthropology 148(2): 276-284; http://dx.doi.org/10.1002/ajpa.21592

Oates, J.F. (2006). Is the Chimpanzee, Pan troglodytes, an endangered species? It depends on what "endangered" means. Primates 47(1): 102-112; http://dx.doi.org/10.1007/s10329-005-0149-5

Oates, J.F., C.P. Groves \& P.D. Jenkins (2009). The type locality of Pan troglodytes vellerosus (Gray, 1862), and implications for the nomenclature of West African chimpanzees. Primates 50(1): 78-80. http://dx.doi.org/10.1007/s10329-008-0116-z

Oates, J.F., C.E.G. Tutin, T. Humle, M.L. Wilson, J.E.M. Baillie, Z. Balmforth, A. Blom, C. Boesch, D. Cox, T. Davenport, A. Dunn, J. Dupain, C. Duvall, C.M. Ellis, K.H. Farmer, S. Gatti, E. Greengrass, J. Hart, I. Herbinger, C. Hicks, K.D. Hunt, S. Kamenya, F. Maisels, J.C. Mitani, J. Moore, B.J. Morgan, D.B. Morgan, M. Nakamura, S. Nixon, A.J. Plumptre, V. Reynolds, E.J. Stokes \& P.D. Walsh (2008). Pan troglodytes. In: IUCN 2013. IUCN Red List of Threatened Species. Version 2013.2. <www.iucnredlist.org>. Downloaded on 15 April 2014

Posada, D. \& K.A. Crandall (2001). Selecting the best-fit model of nucleotide substitution. Systematic Biology 50(4): 580-601; http:// dx.doi.org/10.1080/10635150118469

Perry, G.H., P. Melsted, J.C. Marioni, Y. Wang, R. Bainer, J.K. Pickrell, K. Michelini, S. Zehr, A.D. Yoder, M. Stephens, J.K. Pritchard \& Y. Gilad (2012). Comparative RNA sequencing reveals substantial genetic variation in endangered primates. Genome Research 22(4): 602-610.

Stone, A.C., R.C. Griffiths, S.L. Zegura \& M.F. Hammer (2002). High levels of Y-chromosome nucleotide diversity in the genus Pan. Proceedings of the National Academy of Sciences 99(1): 43-48; http://dx.doi.org/10.1073/pnas.012364999

Stone, A.C., F.U. Battistuzzi, L.S. Kubatko, G.H.J. Perry, E. Trudeau, H. Lin \& S. Kumar (2010). More reliable estimates of divergence times in Pan using complete mtDNA sequences and accounting for population structure. Philosophical Transactions of the Royal Society 365: 3277-3288; http://dx.doi.org/10.1098/rstb.2010.0096

Tamura, K., G. Stecher, D. Peterson, A. Filipski, \& S. Kumar (2013). MEGA6: Molecular Evolutionary Genetics Analysis version 6.0. Molecular Biology and Evolution30: 2725-2729 http://dx.doi. org $/ 10.1093 / \mathrm{molbev} / \mathrm{mst197}$ 